\title{
$\mathrm{BMJ}$
}

\section{Screening versus routine practice in detection of atrial fibrillation in patients aged 65 or over: cluster randomised controlled trial}

\author{
David A Fitzmaurice, professor of primary care, ${ }^{1} \mathrm{~F}$ D Richard Hobbs, professor, head of department, ${ }^{1}$ \\ Sue Jowett, research fellow, ${ }^{1}$ Jonathon Mant, reader, ${ }^{1}$ Ellen T Murray, research fellow, ${ }^{1}$ Roger Holder, head of \\ statistics, ${ }^{1}$ J P Raftery, professor of health technology assessment, ${ }^{2}$ S Bryan, professor of health economics, ${ }^{3}$ \\ Michael Davies, consultant cardiologist, ${ }^{4}$ Gregory Y H Lip, professor of cardiovascular medicine, ${ }^{5}$ \\ T F Allan, senior lecturer ${ }^{6}$
}

\begin{abstract}
Department of Primary Care and General Practice, University of Birmingham, Birmingham B15 2TT

${ }^{2}$ Wessex Institute for Health Research and Development, University of Southampton

${ }^{3}$ Health Economics Facility, University of Birmingham

${ }^{4}$ Selly Oak Hospital, Birmingham

${ }^{5}$ University Department of

Medicine, City Hospital,

Birmingham

${ }^{6}$ Health Care Research Unit,

St Bartholomew's School of

Nursing and Midwifery,

City University, London
\end{abstract}

Correspondence to: F D R Hobbs

f.d.r.hobbs@bham.ac.uk

doi:10.1136/bmj.39280.660567.55

\section{ABSTRACT}

Objectives To assess whether screening improves the detection of atrial fibrillation (cluster randomisation) and to compare systematic and opportunistic screening. Design Multicentred cluster randomised controlled trial, with subsidiary trial embedded within the intervention arm.

Setting 50 primary care centres in England, with further individual randomisation of patients in the intervention practices.

Participants 14802 patients aged 65 or over in 25 intervention and 25 control practices.

Interventions Patients in intervention practices were randomly allocated to systematic screening (invitation for electrocardiography) or opportunistic screening (pulse taking and invitation for electrocardiography if the pulse was irregular). Screening took place over 12 months in each practice from October 2001 to February 2003. No active screening took place in control practices.

Main outcome measure Newly identified atrial fibrillation. Results The detection rate of new cases of atrial fibrillation was $1.63 \%$ a year in the intervention practices and $1.04 \%$ in control practices (difference $0.59 \%, 95 \%$ confidence interval $0.20 \%$ to $0.98 \%$ ). Systematic and opportunistic screening detected similar numbers of new cases $(1.62 \% \vee 1.64 \%$, difference $0.02 \%,-0.5 \%$ to $0.5 \%)$.

Conclusion Active screening for atrial fibrillation detects additional cases over current practice. The preferred method of screening in patients aged 65 or over in primary care is opportunistic pulse taking with follow-up electrocardiography.

Trial registration Current Controlled Trials ISRCTN19633732.

\section{INTRODUCTION}

Atrial fibrillation is a major risk factor for thromboembolic disease, particularly stroke. ${ }^{1}$ Its prevalence rises with age, from about $1 \%$ in the whole population to about $5 \%$ in people aged over $65 .^{2}$ It can be diagnosed by means of a simple low cost test (electrocardiography), which is acceptable to most patients. The risk of serious sequelae, such as stroke, can be dramatically reduced by treatment, with a $68 \%$ relative risk reduction for ischaemic stroke and $25 \%$ reduction in relative mortality associated with warfarin therapy compared with placebo. ${ }^{3}$ Atrial fibrillation therefore fulfils many of the Wilson-Junger criteria for a screening programme. ${ }^{4}$

Two potential population screening strategies include opportunistic case finding and systematic screening. In opportunistic case finding, a healthcare professional would take a patient's pulse during a consultation. If the pulse was irregular electrocardiography would be performed as a confirmatory test as an irregular pulse is a non-specific predictor of atrial fibrillation. ${ }^{5}$ In systematic screening, the whole target population would be invited for screening by electrocardiography.

One study in the United Kingdom compared systematic screening led by nurses with prompted opportunistic case finding for atrial fibrillation in primary care (four practices, $\mathrm{n}=3001$ ). The systematic screening detected more cases than opportunistic case finding, but atrial fibrillation had already been diagnosed in many of the participants and the screening period was only six months, making its generalisability unclear. ${ }^{6}$ Two further studies based in single practices investigated the role of practice nurses in the screening process $^{7}$ and whole population screening ${ }^{8}$ : the first study suggested that opportunistic screening with the pulse taken by a nurse and 12 lead electrocardiography was the most effective screening strategy, while the second study investigated only population screening. It therefore remains unclear which, if any, screening strategy should be adopted.

As part of the screening for atrial fibrillation in the elderly (SAFE) study, we tested whether screening was more effective than routine care in detecting atrial fibrillation in the community and, in a subsidiary randomisation within the screening arm, compared 
opportunistic screening with total population screening.

\section{METHODS}

\section{Design and setting}

This was a multicentred cluster randomised controlled trial (figure) of computerised general practices in England, with individual randomisation of patients in intervention practices to two different screening strategies.

\section{Participants}

We recruited 50 general practices from the Midlands Research Practices Consortium (MidReC). All patients aged 65 or over from these practices were eligible for participation in the study, though patients could be excluded if their own general practitioner thought participation inadvisable.

\section{Randomisation and sequence generation Main trial}

After stratification for practice size and deprivation (based on Townsend score), we used MINITAB to select randomly two equal size groups from those practices within a particular stratum. We used a simulated value from a Bernoulli distribution, comprising two

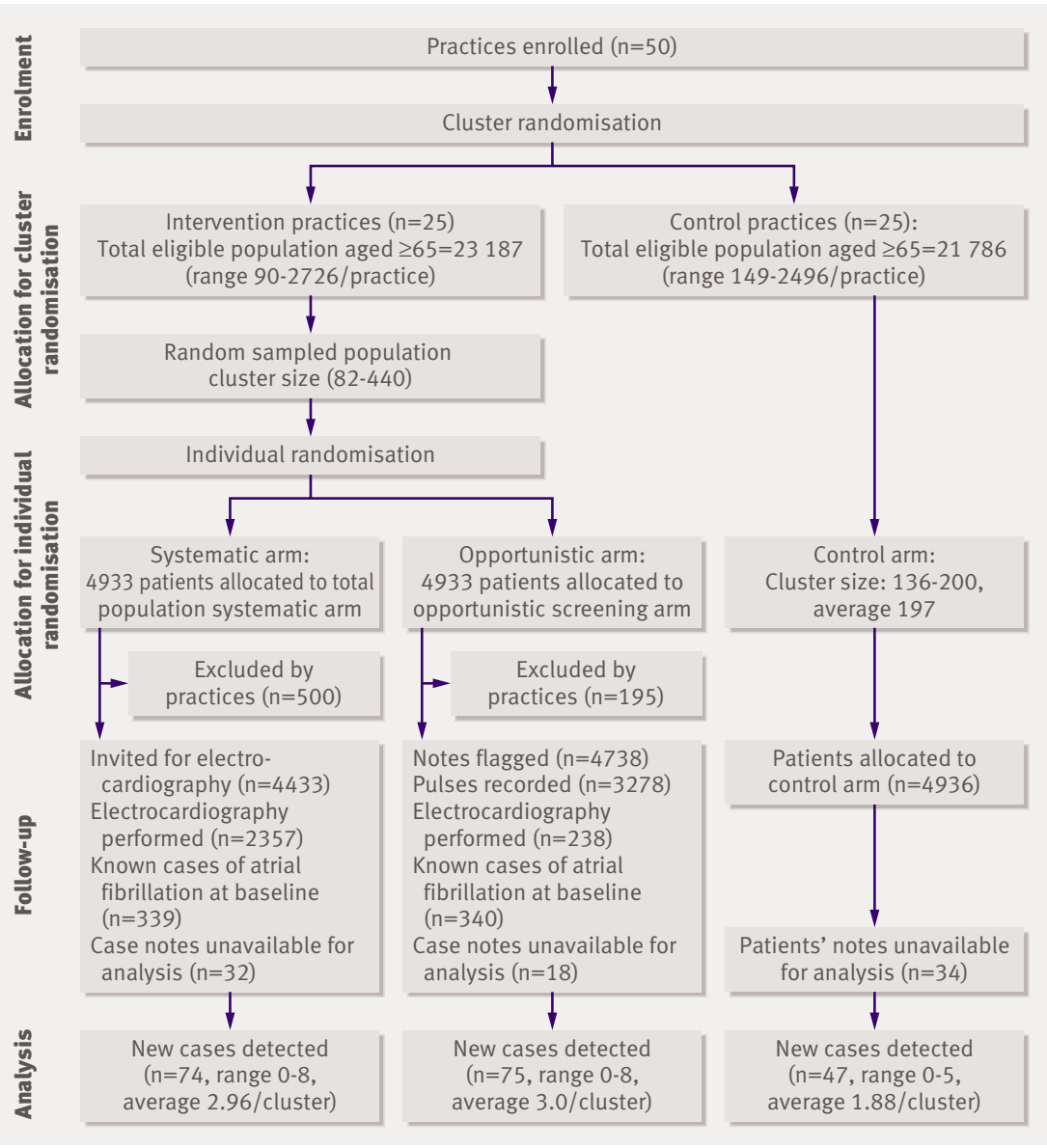

Distribution of practices and participants through stages of study values equally likely to occur, to determine which group became the intervention arm (the other being the control arm).

\section{Subsidiary trial}

After stratification for known atrial fibrillation (see below) we randomly allocated patients from the 25 intervention practices to systematic or opportunistic screening.

We obtained a computerised list of all patients aged 65 or over from each practice. From these we intended to identify random samples of 400 patients from each intervention practice, and 200 from each control practice. As not all practices had as many as 400 eligible patients we selected 440 patients from practices with more than 900 eligible patients, 420 patients from practices with between 420 and 900 eligible patients, and all eligible patients from practices with fewer than 420 eligible patients. Random selection of patients for each practice was achieved with computer generated random numbers (MINITAB) to access entries in lists of patients. For control practices only one practice did not contribute 200 patients and no adjustment was made.

After initial sampling, we returned the lists of patients to the practices and asked them to remove the names of any patients who had died, moved, or were terminally ill. These were replaced by a further random selection of patients from a reserve list of $10 \%$ drawn up for each practice. The cleaned lists from each practice were classified into strata on the basis of whether or not atrial fibrillation had been previously diagnosed (see below). We used SPSS to allocate patients randomly from this list to either systematic or opportunistic screening to create two equal size groups of patients within each stratum so that each strategy (systematic or opportunistic screening) had an equal chance of detecting known, unknown, and suspected atrial fibrillation $(n=4933)$. Which group then became the systematic arm (the other being opportunistic) was again decided by using a simulated value from a Bernoulli distribution, comprising two values equally likely to occur.

Before we flagged notes or invited patients for electrocardiography, the general practitioners scrutinised practice lists for a second time to remove any names of patients who had died, moved, or were terminally ill, and these patients were not replaced.

\section{Blocking}

We did not use explicit blocking in either the main trial or the subsidiary trial but achieved balance at the selection stage by sampling equal numbers from stratified lists of practices and patients.

\section{Allocation concealment}

There was no deliberate concealment of allocation to the trial arms, but the trial management team did not reveal the classification of atrial fibrillation to the participants. The trial statistician determined allocation, which was implemented by the trial coordinator 
Baseline data collection

Identification of atrial fibrillation already known to

practices

We carried out computer searches of practice registers to identify cases of atrial fibrillation in the 15000 study patients using a previously published strategy. ${ }^{9}$ In addition, we identified cohorts of patients receiving prescriptions of digoxin, $\beta$ blockers, class 1,3 , or 4 anti-arrhythmic drugs, aspirin, or warfarin. Clinical records of patients thus identified as having atrial fibrillation or taking drugs that were associated with a diagnosis of atrial fibrillation in any of these computer searches were reviewed for mention of a diagnosis of atrial fibrillation. A diagnosis was accepted if there were hospital letters referring to atrial fibrillation or confirmatory electrocardiograms within the previous five years. We reviewed an additional $5 \%$ random sample of case notes of patients not identified on the computer searches (750 in all) to estimate how many other patients known to have atrial fibrillation were not identified by the computer search strategy.

\section{Intervention}

Primary care physicians and other members of the primary healthcare team in the intervention practices attended investigator days at which they were given educational materials informing them of the importance of detecting atrial fibrillation and the available treatment options. Members of primary healthcare teams from control practices received no educational input.

Practice nurses attended an electrocardiography training day before they started screening clinics. Training included how to perform electrocardiography (with an electronic machine to ensure standardised high quality tracings) and basic interpretation of the electrocardiogram (specifically how to identify atrial fibrillation). Screening was undertaken in phases because of the large number of practices, with the first practice starting screening in October 2001 and the last practice finishing screening in February 2003, with each practice undertaking 12 months of screening.

All patients allocated to systematic screening (including those with known atrial fibrillation) were invited by post to attend a screening clinic. The notes of patients in the opportunistic arm (including those with known atrial fibrillation) were flagged with either a manual paper flag or computer flag to encourage pulse recording during routine consultation. Patients with an irregular pulse were invited to attend a screening clinic. Once this process had been undertaken, the flag was removed from the notes and returned to the research team.

Practice nurses at the screening clinic obtained consent from patients. Thus only those patients who responded to the invitation in the systematic arm $(n=2357)$ and those with an irregular pulse in the opportunistic arm $(n=238)$ provided informed consent. No data were available for those who failed to respond and therefore did not give consent. All data relating to patients were anonymised before they left the general practice, and all research staff held honorary contracts with the participating centres.

Practice nurses ran the screening clinics and collected baseline information and medical history (including any previous diagnosis of atrial fibrillation), took the radial pulse, and carried out 12 lead electrocardiography (Biolog, Numed, Sheffield, UK). Two consultant cardiologists (GL, MD), who were blinded to allocation, assessed whether the electrocardiogram showed atrial fibrillation or not and identified any other relevant abnormalities. A third blinded cardiologist arbitrated on any disagreements over diagnosis. Patients with normal findings on electrocardiography were informed within two weeks and patients with any abnormality were asked to make an appointment with their general practitioner.

\section{Main outcome measures}

Our primary outcome measure was the number of new cases of atrial fibrillation detected during the 12 month study period (newly identified atrial fibrillation). To detect new cases in control practices we carried out computer searches of patients' notes at baseline (see above) and after 12 months of screening. We reviewed case notes of patients identified in these computer searches for evidence of atrial fibrillation (using the same criteria as above).

We assessed acceptability to patients using an adapted version of the screening specific tool used in the colorectal screening programme. ${ }^{10}$ All patients screened were asked to complete an acceptability questionnaire immediately after screening.

\section{Sample size}

We assumed that the baseline prevalence of atrial fibrillation known to the practice would be $3 \%$ (half the expected prevalence of $6 \%{ }^{11}$ ) and that this prevalence would remain constant within the control

\begin{tabular}{|c|c|c|c|c|c|}
\hline & \multirow[b]{2}{*}{ All } & \multirow[b]{2}{*}{ Control } & \multicolumn{3}{|c|}{ Intervention } \\
\hline & & & Total & Opportunistic & Systematic \\
\hline No of patients & 14802 & 4936 & 9866 & 4933 & 4933 \\
\hline Men (\%) & $6302(42.6)$ & $2079(42.1)$ & $4223(42.8)$ & $2104(42.7)$ & $2119(43.0)$ \\
\hline Mean age (SD) & $75.3(7.2)$ & $75.5(7.2)$ & $75.2(7.2)$ & $75.1(7.1)$ & $75.2(7.3)$ \\
\hline Median age & 74.1 & 74.5 & 73.9 & 74.0 & 73.8 \\
\hline Age $<75$ (\%) & $8059(54.4)$ & $2597(52.6)$ & $5465(55.4)$ & $2755(55.8)$ & $2710(54.9)$ \\
\hline
\end{tabular}




\begin{tabular}{|c|c|c|c|c|c|c|}
\hline Group & Patients & Baseline AF & Missing notes & Denominator & $\begin{array}{l}\text { Newly identified } \\
\text { cases }\end{array}$ & New case detection \% \\
\hline Control & 4936 & 389 & 34 & 4513 & 47 & 1.04 \\
\hline \multicolumn{7}{|l|}{ Intervention: } \\
\hline Total & 9866 & 679 & 50 & 9137 & 149 & 1.63 \\
\hline Opportunistic* & 4933 & 340 & 18 & 4575 & 75 & 1.64 \\
\hline Systematic* & 4933 & 339 & 32 & 4562 & 74 & 1.62 \\
\hline
\end{tabular}

practices. To have $90 \%$ power at a significance level of $5 \%$ to detect a $1 \%$ difference in the detection rate of new cases of atrial fibrillation between the different arms of the study we required 5000 patients in 25 practices (cluster size 200) in each group. This assumed an intracluster correlation coefficient of 0.015 (derived from prevalence data from a previous study). ${ }^{11}$

\section{Statistical methods}

We analysed data on an intention to treat basis. We subtracted any previously known cases of atrial fibrillation from the totals obtained at the end of the study to prevent double counting in the newly identified atrial fibrillation figures. Proportions and rates were used as the measures of prevalence and newly identified atrial fibrillation. We used $\chi^{2}$ test to compare overall 12 month prevalence and incidence rates between arms.

\section{Incidence analysis}

We used non-linear mixed models with binomial error to examine variation from arm to arm in the rates of newly identified atrial fibrillation within the total population minus the population with known atrial fibrillation ( $\mathrm{n}=13734)$, allowing for age (modelled as above or below the median age (73.66) of those patients within this reduced population) and sex and including both main effects and interaction terms. Practice was defined as a random effect to account for extra binomial variability at the practice level. Any difference in baseline prevalence at practice level was accounted for with the inclusion of the log odds of baseline atrial fibrillation. These analyses were conducted using Proc Nlmixed in SAS (version 8.2) (SAS, Carey, NC). The analysis was repeated without the inclusion of the covariate describing baseline prevalence.

We used logistic regression to compare rates of newly identified atrial fibrillation per practice in the control and intervention arms and also in a matched pairs analysis of 12 month incidence between the opportunistic and systematic arms.

\section{RESULTS}

\section{Participant flow}

Table 1 gives details of age and sex of the study participants by screening arm. We reviewed case notes of $5216(35 \%)$ of the total study population at baseline because they had been picked up on computer search as having atrial fibrillation or relevant drug code.

\section{Baseline prevalence of atrial fibrillation}

We confirmed atrial fibrillation in 1068 patients (20.5\% of search positive population, and $7.3 \%$ of total population) by reviewing clinical records. Case notes were unavailable for 21 patients. In the additional random 750 sets of case notes that we reviewed as a validation of the computer searches, we found no extra cases of atrial fibrillation. The baseline prevalence was $7.9 \%$ (95\% confidence interval $7.2 \%$ to $8.7 \%)$ in the control arm and $6.9 \%(6.2$ to $7.6 \%)$ in both the opportunistic and systematic arms. The intraclass correlation was 0.0027 across all practices.

\section{Newly identified atrial fibrillation over 12 months}

Table 2 shows the number of new cases of atrial fibrillation found through searching notes at 12 months after the baseline search. We calculated rates of newly identified atrial fibrillation after removing those patients in whom atrial fibrillation was diagnosed at baseline and patients whose notes could not be found. Newly identified atrial fibrillation therefore refers to the number of new cases diagnosed within the 12 month study period. Data were missing for patients whose notes were no longer available because of death or no longer being at the practice. The overall mean 12 month prevalence was $8.9 \%(7.9 \%$ to $9.7 \%)$ in the control arm, $8.4 \%(7.6 \%$ to $9.4 \%)$ in the opportunistic arm, and $8.4 \%(7.6 \%$ to $9.3 \%)$ in the systematic arm.

The detection rate of new cases of atrial fibrillation was $1.63 \%$ a year in the intervention practices and $1.04 \%$ in control practices (difference 0.59\%, 0.20\% to 0.98). Systematic and opportunistic screening detected similar new numbers of cases $(1.62 \%$ and $1.64 \%$; difference $0.02 \%,-0.5 \%$ to $0.5 \%$ ) (table 2 ).

After we accounted for possible practice to practice variation using a mixed model with binomial error variation, we found a significant difference in the detection of new cases of atrial fibrillation between the control arm and the opportunistic and systematic arms $(\mathrm{P}=0.01$ and $\mathrm{P}=0.02$, respectively) but not between the opportunistic and systematic arms $(\mathrm{P}=0.95)$. Within each of the three arms there was no significant practice to practice variation $(\mathrm{P}=0.88, \mathrm{P}=0.62$, and $\mathrm{P}=0.96$, respectively). After we accounted for baseline prevalence, screening practices identified substantially more cases of atrial fibrillation than the control practices (odds ratio $1.61,1.14$ to $2.29, \mathrm{P}=0.0085$ ), and we obtained similar results when we removed baseline prevalence from the model $(1.58,1.12$ to 2.22 , 
$\mathrm{P}=0.0103)$. A matched pairs logistic regression analysis between patients from the same practices allocated to either the opportunistic or systematic arms showed no significant difference in detection of new cases of atrial fibrillation at 12 months $(\mathrm{P}=0.67)$ or between practices $(\mathrm{P}=0.60)$.

\section{Opportunistic $v$ systematic screening}

We identified 75 new cases of atrial fibrillation in the opportunistic screening group and 74 new cases in the systematic screening group. Differences in screening method had no effect on the rate of detection between the groups (odds ratio $0.99,0.72$ to $1.37, \mathrm{P}=0.95$ ).

In total $3278(69 \%)$ patients were opportunistically screened for atrial fibrillation during the 12 months of the study. After they had had their pulse taken, 122/ $360(34 \%)$ patients with an irregular pulse declined electrocardiography. Of the remaining patients, atrial fibrillation was diagnosed in $84 / 238(35 \%)$ on electrocardiography $(2.6 \%$ of patients who had their pulse taken). Of patients who had an irregular pulse but no electrocardiography, atrial fibrillation had already been confirmed in 56/122 (46\%). Of the 75 new cases of atrial fibrillation in the opportunistic arm, 24 had a regular pulse recorded but subsequently had atrial fibrillation diagnosed outside the screening programme. The irregular pulse could have been missed in these 24 patients; or they could have developed atrial fibrillation subsequent after they had their pulse taken; or they may have had paroxysmal atrial fibrillation. We cannot distinguish between these three possibilities.

Of the 4433 patients invited for systematic screening, 2357 (53\%) underwent electrocardiography, 904 $(20 \%)$ declined screening, and $992(22 \%)$ did not respond. Some $135(6 \%)$ were found to have atrial fibrillation, which was a new diagnosis in 52 (39\%) (the 22 additional cases were detected outside the screening programme).

\section{Prevalence of paroxysmal atrial fibrillation}

Of the 339 intervention patients confirmed to have atrial fibrillation at baseline and randomised to systematic screening, 205 underwent screening electrocardiography during the study, which confirmed atrial fibrillation in $136(66 \%)$. The electrocardiogram showed sinus rhythm in 69 (34\%). Thus, about a third of prevalent atrial fibrillation at baseline was either self limiting or paroxysmal.

All patients undergoing electrocardiography $(\mathrm{n}=2595)$ were given a questionnaire after screening, of which 1940 (75\%) were returned completed. Most (95\%) respondents thought that screening was important and that sufficient information was available before screening.

\section{DISCUSSION}

In this multicentred study we have identified the most effective manner of screening for atrial fibrillation in patients aged 65 or over. Opportunistic screening with opportunistic pulse taking, prompted by flagged case notes, during routine clinic attendances and then performing 12 lead electrocardiography on those with an irregular pulse was as effective as systematic population screening, with both being more effective than "routine practice," which comprised non-systematic case finding. This finding suggests that routine electrocardiography within this population is unnecessary for the detection of atrial fibrillation as long as healthcare professionals are conscientious about feeling the pulse.

The utility of opportunistic screening was undoubtedly due in part to the extensive coverage attained over the 12 month study period (69\%) in contrast to a lower coverage rate $(29 \%)$ over a six month period in the Southampton study. ${ }^{6}$ Within SAFE opportunistic pulse taking was encouraged by the flagging of clinical notes. This was predominantly manual, though with the increase in the use of wholly computerised records, and improvements in practice administration driven by incentives, such as the quality and outcomes framework in the UK, the potential for opportunistic screening will probably increase. The validity of our findings may be transferable to any system of managed care where high rates of coverage can be achieved.

There was no significant difference in the mean community prevalence of atrial fibrillation at 12 months

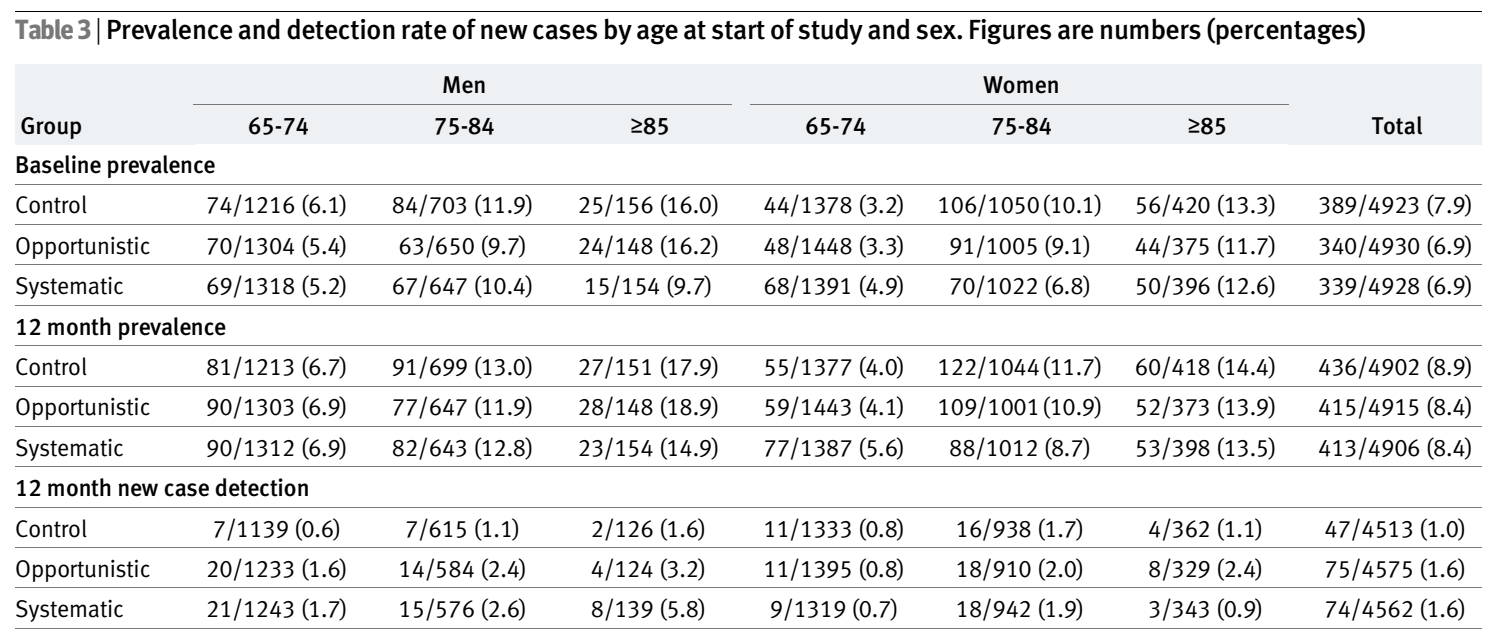




\section{WHAT IS ALREADY KNOWN ON THIS TOPIC}

Atrial fibrillation is a major modifiable risk factor for stroke

Atrial fibrillation is underdiagnosed and undertreated in the community

\section{WHAT THIS STUDY ADDS}

Screening in general practice will identify new cases of atrial fibrillation

Opportunistic screening with pulse taking followed by electrocardiography is as effective as systematic screening of patients aged 65 or over

between the three arms of the study. The baseline prevalence was $6.9 \%-7.9 \%$ in those aged 65 or over. The SAFE prevalence rates of atrial fibrillation represent more robust estimates than the previously reported prevalence in this population of $4.5 \%$ from systematic screening $^{6}$ and $5.4 \%$ from total population screening. ${ }^{8}$ Routinely reported data for those aged 85 or over has suggested a prevalence of atrial fibrillation of around $10 \%,{ }^{2}$ significantly lower than we found in our study. These earlier studies were small and in less investigated populations and probably systematically underreported atrial fibrillation, whereas the SAFE population was, by design, much larger and more representative than earlier studies with more robust methods of screening. General practitioners removed more patients from the opportunistic screening arm than from the systematic screening arm, although the same criteria were used for removal. This discrepancy is relatively small and does not affect our main outcome.

Little information is available on the rate of newly identified atrial fibrillation. One small Scottish study in younger people (45-64) reported a four year incidence of 0.54 per 1000 patient years. ${ }^{12}$ In this study there were also significant differences between the populations, with the detection rate being significantly higher in screened populations than in routine practice.

The prevalence and incidence rates for atrial fibrillation in SAFE are therefore likely to represent reliable estimates for the UK (table 3). The screening processes, whether systematic or opportunistic, did not raise anxiety and were acceptable to patients. As the detection rates were essentially identical for the two methods, however, the more labour intensive, costly, and intrusive approach with systematic screening cannot be justified. Furthermore, systematic screening was much less acceptable to patients overall, with over $20 \%$ positively declining the offer and over $26 \%$ not responding to the offer.

\section{Limitations of study}

Our study was complicated by the fact that the control population had a higher prevalence of atrial fibrillation at baseline, which we assumed to be a random finding. This had to be corrected for in the analysis. Further study limitations included the inability to detect all cases of paroxysmal atrial fibrillation (although this was presumed in patients whose case notes indicated atrial fibrillation but whose electrocardiograms were negative) and the poor uptake of systematic screening. These data do not directly tell us how often screening should take place, though this will be estimated in future economic modelling of the clinical yields on the basis of the observed new detection rates. We will also model the effect of antithrombotic treatment on the incident cases. This will affect the cost effectiveness of any screening programme, given that the earlier atrial fibrillation is detected the sooner antithrombotic treatment can start. It is not clear how uptake in patients would be affected if this became a routine procedure. Patients may have been reluctant to participate in a research project as opposed to undertaking a routine screen. Uptake of cervical screening, for example, improved dramatically over the past decade as it became routine. $^{13}$

Contributors: DAF and FDRH were principal investigators. SJ was research fellow for the project. JM was joint grant holder. ETM was project manager. RH was head of statistics. JPR was joint grant holder and contributed to study design. SB was principal health economist for the study. MD and GYHL were joint grant holders and read the electrocardiograms. TFA was joint grant holder and study statistician. DAF, FDRH, SJ, JM, and RH wrote the paper. DAF is guarantor.

Funding: NHS research and development health technology assessment programme (No 96/22/11).

Competing interests: None declared.

Ethical approval: West Midlands multicentre research ethical committee. Provenance and peer review: Non-commissioned, externally peer reviewed.

1 Wolf PA, Abbott RD, Kannel WB. Atrial fibrillation as an independent risk factor for stroke: the Framingham study. Stroke 1991;22:983-8.

2 Majeed A, Moser K, Carroll K. Trends in prevalence of atrial fibrillation in general practice in England and Wales, 1994-1998: analysis of data from the general practice research database. Heart 2001;86:284-8.

3 Atrial Fibrillation Investigators. Risk factors for stroke and efficacy of antithrombotic therapy in atrial fibrillation; analysis of pooled data from five randomised controlled trials. Arch Int Med 1994;154:1449-57.

4 Wilson JMG, Jungner G. The principles and practice of screening for disease. WHO Public Health papers. Geneva: World Health Organization, 1968:34.

5 Sudlow M, Rodgers H, Kenny RA, Thomson R. Identification of patients with atrial fibrillation in general practice: a study of screening methods. BMJ 1998;317:327-8.

6 Morgan S, Mant D. Randomised trial of two approaches to screening for atrial fibrillation in UK general practice. Br J Gen Pract 2002;52:373-80.

7 Somerville S, Somerville J, Croft P, Lewis M. Atrial fibrillation: a comparison of methods to identify cases in general practice. Br J Gen Pract 2000;50:727-9.

8 Wheeldon NM, Tayler DI, Anagnostou E, Cook D, Wales C, Oakley GDG. Screening for atrial fibrillation in primary care. Heart 1998;79:50-5

9 Lip GYH, Golding D, Nazir M, Beevers D, Child D, Fletcher R. A survey of atrial fibrillation in general practice: the West Birmingham atrial fibrillation project. BrJ Gen Pract 1997;47:285-9.

10 Marjoram I, Strachan R, Allan A, Allan E. Screening for colorectal cancer a general practice based study. BrJ Gen Pract 1996;46:283-6.

11 Davies RC, Hobbs FDR, Kenkre JE, Roalfe AK, Hare R, Lancashire RJ, Davies MK. Prevalence of left ventricular systolic dysfunction and heart failure in high risk patients: community based epidemiological study. BMJ 2002;325:1156-61.

12 Stewart S, Hart CL, Hole DJ, McMurray JJ. Population prevalence, incidence, and predictors of atrial fibrillation in the Renfrew/Paisley study. Heart 2001;86:516-21.

13 Onyeka BA. Martin-Hirsch P. Information leaflets, verbal information and women's knowledge of abnormal cervical smears and colposcopy. J Obstet Gynaecol 2003;23:174-6.

Accepted: 29 June 2007 